\title{
Magnetic characterization of nanocrystalline iron samples with different size distributions
}

\author{
Janusz TyPeK ${ }^{1 *}$, Grzegorz Zolnierkiewicz ${ }^{1}$, RAfal Pelka ${ }^{2}$, Karolina Kielbasa $^{2}$, \\ WALERIAN ARABCZYK ${ }^{2}$, NIKOS GUSKOS ${ }^{1,3}$ \\ ${ }^{1}$ Institute of Physics, West Pomeranian University of Technology, 48 Piastow Str., 70-311 Szczecin, Poland \\ ${ }^{2}$ Institute of Chemical and Environment Engineering, West Pomeranian University of Technology \\ 10 Pulaskiego Str., 70-322 Szczecin, Poland \\ ${ }^{3}$ Department of Solid State Physics, Faculty of Physics, University of Athens, Panepistimiopolis, \\ 15784 Zografos, Athens, Greece
}

\begin{abstract}
Nanocrystalline iron was obtained by fusing magnetite and promoters. The oxidized form was reduced with hydrogen and passivated (sample P0). The average nanocrystallite size in sample $\mathrm{P} 0$ was $d(\mathrm{P} 0)=16 \mathrm{~nm}$ and the width of size distribution was $\sigma(\mathrm{P} 0)=18 \mathrm{~nm}$. Samples of nanocrystalline iron with narrower diameter ranges and larger and smaller average crystallite sizes were also synthesized. They were: sample $\mathrm{P} 1(d(\mathrm{P} 1)=28 \mathrm{~nm}, \sigma(\mathrm{P} 1)=5 \mathrm{~nm})$, sample $\mathrm{P} 2(d(\mathrm{P} 2)=22 \mathrm{~nm}, \sigma(\mathrm{P} 1)=$ $5 \mathrm{~nm})$, sample $\mathrm{P} 3(d(\mathrm{P} 3)=12 \mathrm{~nm}, \sigma(\mathrm{P} 1)=9 \mathrm{~nm})$. These four samples were studied at room temperature by dc magnetization measurements and ferromagnetic resonance at microwave frequency. Correlations between samples sizes distributions (average size and width of the sizes) and magnetic parameters (effective magnetization, anisotropy field, anisotropy constant, FMR linewidth) were investigated. It was found that the anisotropy field and effective magnetization determined from FMR spectra scale linearly with nanoparticle sizes, while the effective magnetic anisotropy constant determined from the hysteresis loops decreases with nanoparticle size increase.
\end{abstract}

Keywords: nanocrystalline iron; ferromagnetic resonance; magnetic property

(C) Wroclaw University of Technology.

\section{Introduction}

Nanocrystalline materials have high surface area to volume ratio compared to the coarsegrained substances, fewer localized defects (dislocations, vacancies), and in the area of grain boundaries, atoms of higher energy states are localized. Compared to coarse-grained materials they also have greater mechanical strength, good adhesion to a support, hardness, and corrosion resistance. As many of these properties depend critically on the crystallite sizes [1-4] methods for the separation of fractions of material containing specified crystallite sizes are needed.

For this purpose a multistep method of separation was developed and applied to nanocrystalline iron [5]. Firstly, the distribution of crys-

*E-mail: typ22jan@zut.edu.pl tallites was measured by known methods, for example by Warren Averbach X-ray diffraction, by electron microscopy or by the methods described elsewhere [6,7]. Nanocrystalline iron was then subjected to an oxidation process with water vapor. The reaction was continued until a desired degree of conversion, equivalent to the crystallite diameter $d_{1}$, depending on the size of the crystallites, which were intended to be extracted. Stopping the reaction at an appropriate stage, a mixture of two phases - an oxide phase and a metal phase - was obtained. Two-phase mixture was subjected to a leaching process in diluted acid. The result was the dissolution of iron crystallites. At this stage nanomaterial in the form of iron oxide with a specific, predetermined size of crystallites was obtained. Subsequently, the resulting material was subjected to reduction process again until the conversion degree was equivalent to the average 
diameter of nanocrystallites, $d_{2}$, thus narrowing the distribution of crystallites to the desired size. After successive dissolution of the metal phase and reducing the resulting oxide phase, nanomaterial containing iron crystallites with an average diameter in the range of $d_{1}$ to $d_{2}$ was obtained. Subjecting the obtained nanocrystalline iron with crystallites of a particular size to carburization, nitriding or oxidation reaction, nanocrystalline iron carbides, nitrides or oxides of desired crystallite sizes were obtained.

Ferromagnetic resonance (FMR) is a very useful and convenient method in study of iron nanoparticles [8-10]. Their magnetic characteristics (agglomeration, magnetocrystalline/shape anisotropy, core/shell structure) and dynamics (blocking temperature, dipole-dipole/exchange interactions) could be inferred from FMR spectra study at different temperatures. In this work we study the influence of different size distributions of iron nanoparticles on magnetic characteristics obtained from FMR and magnetization methods.

\section{Experimental}

Nanocrystalline iron was obtained by fusing magnetite together with such oxides as $\mathrm{Al}_{2} \mathrm{O}_{3}, \mathrm{CaO}$ and $\mathrm{K}_{2} \mathrm{O}$ (total amount ca. 7 wt.\%) added to aid in the maintenance of stable structure of small (ca. $20 \mathrm{~nm}$ ) iron nanocrystallites. After cooling and crushing the alloy, a fraction of $1.0-1.2 \mathrm{~mm}$ particles was selected. The oxide form was reduced with hydrogen (flow $20000 \mathrm{~h}^{-1}$ ) in a tubular reactor under atmospheric pressure at $200{ }^{\circ} \mathrm{C}$ - for $1 \mathrm{~h}, 350{ }^{\circ} \mathrm{C}-$ for $2 \mathrm{~h}, 400{ }^{\circ} \mathrm{C}-$ for $12 \mathrm{~h}, 450{ }^{\circ} \mathrm{C}$ - for $24 \mathrm{~h}$ and finally $500{ }^{\circ} \mathrm{C}$ - for $24 \mathrm{~h}$, and then passivated (designated as reference sample P0). Mean size of the crystallites $\left(d_{m}=16 \mathrm{~nm}\right)$ was determined by X-ray diffraction measurements (X-ray diffractometer Philips X'Pert PRO, $\mathrm{CuK} \alpha$, Almelo, the Netherlands) using the Scherrer and Rietveld methods. Specific surface area of catalyst $\left(S=12 \mathrm{~m}^{2} \mathrm{~g}^{-1}\right)$ was determined using a method based on Brunauer-Emmett-Teller (BET) theory (QuadraSorb SI apparatus, Quantachrome Instruments, Boynton Beach, USA). Iron nanocrystallites size distribution was determined by measuring the nitriding reaction rate of the catalyst as described by Pelka et al. [7]. The rate of chemical reactions occurring between gas phase and iron was measured in a tubular reactor making it possible both to measure by thermogravimetry the reaction product content in the solid sample, and to analyze the composition of the gas phase [11]. The gas flow rate at the inlet to the reactor was controlled by means of electronic flowmeters. Gas samples for analysis were collected in the direct vicinity of the catalyst and the hydrogen concentration was determined. It was found that the composition of the gas mixture over and below the catalyst bed was the same. Due to the lack of the gradient of gaseous reactants concentrations in the reaction zone of the reactor and the arrangement of catalyst sample, the reactor can be considered as a differential one. A sample of $1 \mathrm{~g}$ was placed in a form of single layer of grains in a platinum basket, suspended on the arm of a thermobalance. Based on earlier studies [12] it was found that the chemical processes were carried out in the area where the chemical reaction rate was limited by the rate of adsorption of gaseous reactant upon the solid surface. The oxidation process was preceded by reduction of a passive layer of the catalyst and annealing the samples. The catalyst was reduced polythermally to $500{ }^{\circ} \mathrm{C}$ at atmospheric pressure. Hydrogen load was $9 \mathrm{dm}^{3} \mathrm{~h}^{-1} \mathrm{~g}^{-1}$. The sample was next oxidized to magnetite at $500{ }^{\circ} \mathrm{C}$ in a stream of nitrogen $\left(20 \mathrm{dm}^{3} \mathrm{~N}_{2} /(\mathrm{h} \mathrm{g})\right)$ saturated with water vapor $\left(0.02\right.$ bar $\left.\mathrm{H}_{2} \mathrm{O}\right)$ to oxidation degree $\alpha=0.2$. Metallic iron was selectively dissolved with dilute nitric acid solution (V). Magnetite remaining after etching was again reduced with hydrogen. Such samples were then analyzed with respect to magnetic properties.

Magnetic resonance measurements were performed on a conventional Bruker E 500 spectrometer operating in the X-band $(v=9.5 \mathrm{GHz})$ with $100 \mathrm{kHz}$ magnetic field modulation.

FMR spectra of four investigated nanomaterials were registered at room temperature. Magnetization study was performed using a Quantum Design Magnetic Property Measurements System MPMS XL-7 with superconducting quantum 
interference device magnetometer in magnetic fields up to $70 \mathrm{kOe}$.

\section{Results and discussion}

In Fig. 1 exemplary results of XRD measurements are presented for samples designated as P1, P2 and P3, after oxidizing and etching stage of sample preparation. For comparison, data for the reference sample P0 is shown, as well. Phase analysis was based on ICDD PDF2 cards for iron and magnetite, respectively, No. 06-0696 and 88-0315. It was found that at this stage samples $\mathrm{P} 1$ and $\mathrm{P} 2$ contained mainly magnetite $\left(\mathrm{Fe}_{3} \mathrm{O}_{4}\right)$. Sample P3 contained magnetite as well as iron. No impurities were detected. In Fig. 2 size distributions (GSD - grain size distribution) of iron and magnetite nanocrystallites for samples oxidized and etched (samples: P1, P2, P3), and for the reference sample $\mathrm{P} 0$, are presented. These curves are theoretical ones obtained by the method presented by Pelka et al. [7]. Mean size of magnetite nanocrystallites in samples P1 and P2 was, respectively, $28 \mathrm{~nm}$ and $22 \mathrm{~nm}$, however, the value of distribution width $\sigma=5 \mathrm{~nm}$ (defined as peak width at half high) was clearly smaller that for sample P0 $(\sigma=18 \mathrm{~nm})$. It means that the size distribution of nanocrystallites has been narrowed. Mean crystallite size of magnetite and iron contained in sample P3 was ca. $12 \mathrm{~nm}(\sigma=9 \mathrm{~nm})$. It was also found that the etched samples had relatively large surface areas as compared to the reference sample P0: $10-15 \mathrm{~m}^{2} / \mathrm{g}$ (for P1), $20-25 \mathrm{~m}^{2} / \mathrm{g}$ (for P2), $40-50 \mathrm{~m}^{2} / \mathrm{g}$ (for P3). After reduction of iron oxide with hydrogen and passivation, all samples contained metallic iron. It was also experimentally confirmed that the crystallite sizes distributions were unchanged after the reduction. Such samples were then analyzed with respect to magnetic properties.

In Fig. 3 the FMR spectra of investigated samples registered at room temperature and normalized to a unit mass are presented. A very broad, asymmetrical lines typical of agglomerated ferromagnetic nanoparticles can be seen. In comparison with the case of FMR line registered for magnetite nanoparticles it is shifted toward smaller magnetic

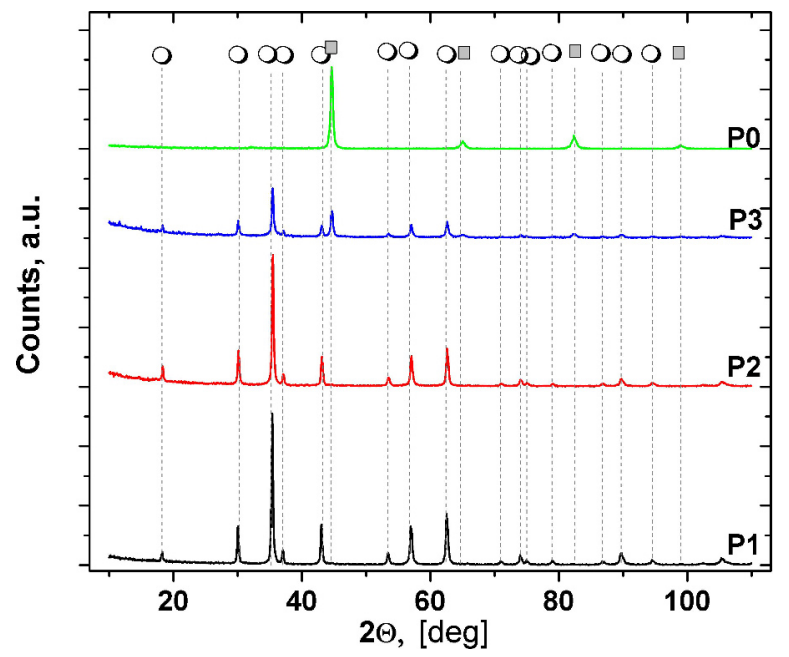

Fig. 1. XRD spectra for samples P0, P1, P2, P3 after etching stage of sample preparation. Main peaks for - iron phase, $\bigcirc$ - magnetite phase are marked based on ICDD PDF2 cards, respectively, No. 06-0696 and 88-0315.

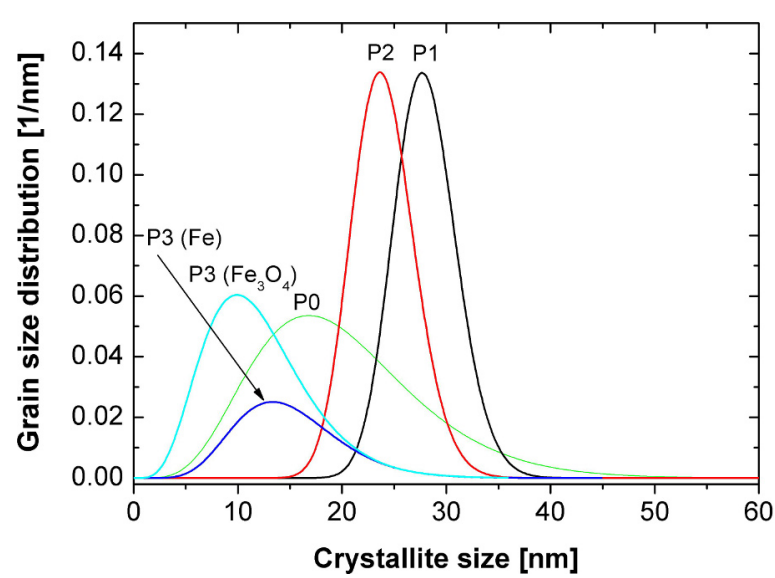

Fig. 2. Crystallite size distributions of samples P1 - P3 after etching stage. Data for the reference sample $\mathrm{P} 0$ is also included.

fields and much broader [13]. The observed FMR spectrum could be regarded as the sum of many individual lines originating from a collection of randomly oriented nanoparticles. As a very crude approximation we have tried to fit the FMR broad line with only a few simpler spectral lines that could be due to the assumed magnetic anisotropy of a nanoparticle or to a different phase present in the investigated sample [14]. In case of samples $\mathrm{P} 1, \mathrm{P} 2$, and P3, a satisfactory agreement with the 
experimental line has been achieved by its decomposition into only two components of Lorentzian line shape. In case of sample P0, at least three components were needed to obtain a reasonable fitting.

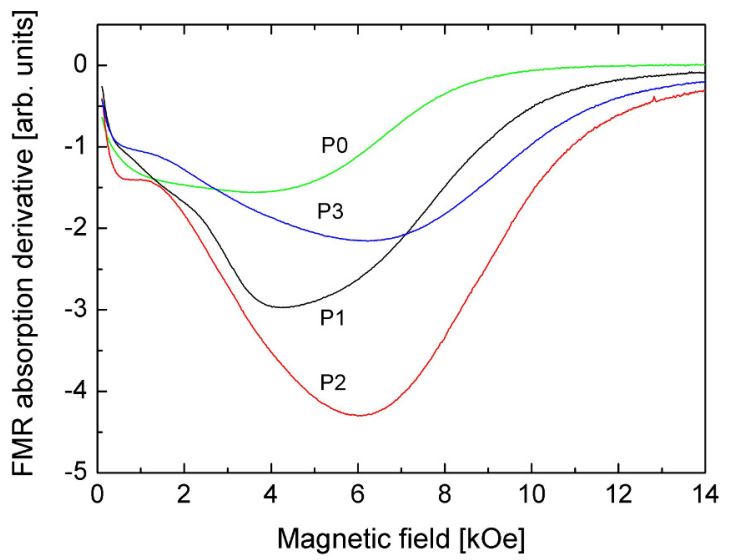

Fig. 3. FMR spectra of investigated samples at room temperatures normalized to a unit mass.

In Table 1 values of the FMR parameters calculated from the decomposition of the experimental spectra into two (or three in case of $\mathrm{P} 0$ sample) are listed. These two components are assumed to arise from magnetic anisotropy and are designated as parallel and perpendicular components. The difference of these resonance fields, $H_{\perp}-H_{\|}$, is proportional to the effective anisotropy field [15]. As could be seen from Table 1, for samples P1, P2 and P3, the anisotropy field scales with the size of a nanoparticle. The same dependence holds for the line widths of the component lines (Fig. 4, left axis). The third, additional component in P0 sample, should be considered separately, as its resonance field is almost at zero magnetic field. It might be attributed to additional phase (magnetite) that was not present in other samples.

The two main components could correspond to the lines originating from parallel and perpendicular orientations of a nanoparticle with respect to an external magnetic field. It might be assumed that the morphology of our samples could be modeled by layers of nanoparticles that surround empty spaces creating sponge-like structure. In this case the magnetic resonance fields in those two orientations could be determined by the Kittel's set of two

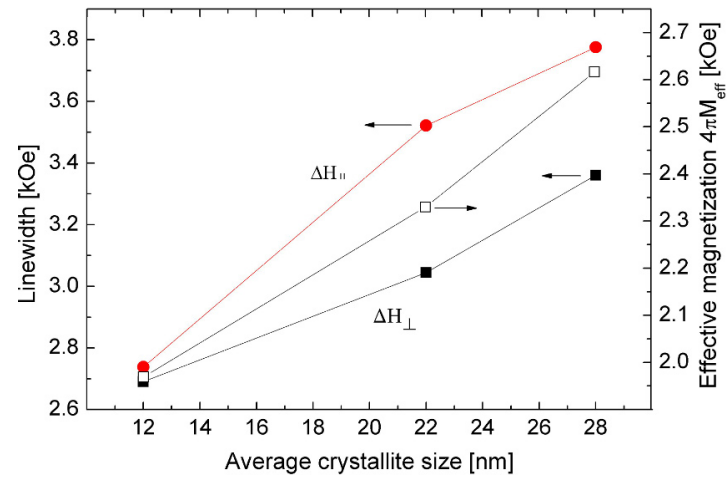

Fig. 4. Dependence of the FMR linewidths (left axis) and the effective magnetization on average magnetization size of nanoparticles (right axis) for P1, P2, and P3 samples.

equations, appropriate for the planar ensembles of nanoparticles [16]:

$$
\begin{gathered}
h v=g_{e f f} \mu_{B}\left(H_{\perp}-4 \pi M_{e f f}\right) \\
h v=g_{e f f} \mu_{B} \sqrt{\left[H_{\|}\left(H_{\|}+4 \pi M_{e f f}\right)\right]}
\end{gathered}
$$

where equation 1 corresponds to the perpendicular and equation 2 to the parallel orientation of the external magnetic field. In the above equations $v$ is the resonance frequency, $\mu_{B}$ is the Bohr magneton, $H_{\perp}$ and $H_{\|}$are the experimental values of magnetic resonance fields, $g_{\text {eff }}$ is the effective spectroscopic $g$-factor and $4 \pi M_{\text {eff }}$ is the effective magnetization of an ensemble of nanoparticles.

Calculated values from equations 1 and 2 of the effective spectroscopic $g$-factor, $g_{e f f}$, and the effective magnetization, $M_{e f f}$, for the investigated samples are collected in Table 2. The effective magnetization is observed to increase with the size of nanoparticles for P1 - P3 samples (Fig. 4, right axis). The saturation magnetization in bulk iron is $M_{S}=1747$ Oe [17].

In Fig. 5 ferromagnetic loops for P1 and P2 samples at room temperature are presented. Left and right insets show the loop in reduced and extended scales, respectively. The saturation magnetization $M_{S}$, obtained for samples $\mathrm{P} 1$ and $\mathrm{P} 2$, is $164 \mathrm{emu} / \mathrm{g}$ and $157 \mathrm{emu} / \mathrm{g}$, respectively. For bulk iron $M_{S}=222 \mathrm{emu} / \mathrm{g}$ [18]. For iron nanoparticles, the values of saturation magnetization smaller 
Table 1. Values of the FMR parameters determined from the decomposition of registered spectra at room temperature.

\begin{tabular}{|c|c|c|c|c|c|}
\hline & & Sample P0 & Sample P1 & Sample P2 & Sample P3 \\
\hline \multirow{3}{*}{ Parallel component } & Amplitude [a.u] & 0.13 & 0.75 & 2.15 & 1.32 \\
\hline & Resonance field [Oe] & 1416 & 1192 & 1543 & 1332 \\
\hline & Linewidth [Oe] & 1965 & 2738 & 3522 & 3776 \\
\hline \multirow{3}{*}{ Perpendicular component } & Amplitude [a.u.] & 0.06 & 0.50 & 0.97 & 0.63 \\
\hline & Resonance field [Oe] & 3800 & 3910 & 4774 & 4910 \\
\hline & Linewidth [Oe] & 1695 & 2690 & 3044 & 3360 \\
\hline \multirow{3}{*}{ Additional component } & Amplitude [a.u.] & 0.18 & - & - & - \\
\hline & Resonance field [Oe] & $\sim 0$ & - & - & - \\
\hline & Linewidth [Oe] & 2604 & - & - & - \\
\hline
\end{tabular}

Table 2. Values of the effective spectroscopic $g$-factor, $g_{e f f}$, and the effective magnetization, $4 \pi M_{e f f}$, at room temperature calculated from equations 1 and 2 .

\begin{tabular}{ccccc}
\hline & Sample P0 & Sample P1 & Sample P2 & Sample P3 \\
\hline \hline$g_{\text {eff }}$ & 3.22 & 3.48 & 2.76 & 2.95 \\
$4 \pi M_{\text {eff }}[\mathrm{kOe}]$ & 1.70 & 1.96 & 2.32 & 2.61 \\
\hline
\end{tabular}

than for bulk iron, due to surface effects, have been reported [17, 19]. Remnant magnetization $M_{r}$ is equal to $31 \mathrm{emu} / \mathrm{g}\left(M_{r} / M_{S}=0.19\right)$ and $41 \mathrm{emu} / \mathrm{g}$ $\left(M_{r} / M_{S}=0.26\right)$ for $\mathrm{P} 1$ and $\mathrm{P} 2$ samples, respectively. The values of coercive field $H_{c}$ for P1 and P2 samples are 146 Oe and 240 Oe, respectively.

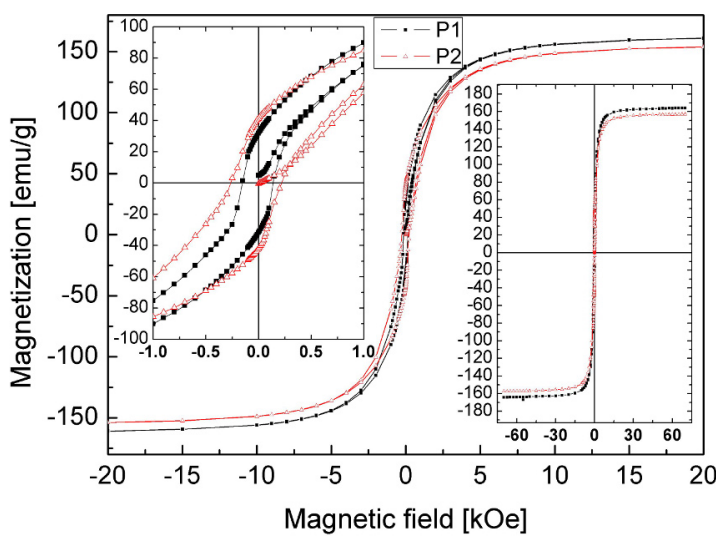

Fig. 5. Ferromagnetic loops for $\mathrm{P} 1$ and $\mathrm{P} 2$ samples registered at room temperature. Left and right insets show the loops in reduced and extended scales, respectively.
For an assembly of non-interacting 3D random particles the Stoner-Wohlfarth model predicts the following expressions: $H_{c}=0.958 K_{1} / M_{s}$, $M_{r} / M_{s}=0.5$ for uniaxial anisotropy and $H_{c}=0.64$ $K_{1} / M_{s}, M_{r} / M_{s}=0.832$ for cubic anisotropy [18]. In the above expressions $K_{1}$ is the anisotropy constant, equal to $5 \cdot 10^{5} \mathrm{erg} / \mathrm{cc}$ for bulk Fe. However, this theory does not explain magnetic properties of real nanoparticles. Experiments with $\mathrm{Fe}$ particles having different selected diameters in the range of $3 \div 40 \mathrm{~nm}$ have shown that the ratio $M_{r} / M_{S}<0.40$ slowly increases with the particle size [20]. Thus, this ratio is almost equal to that for uniaxial anisotropy in spite of cubic crystal symmetry of Fe. The coercive field was found to increase with nanoparticle size what agrees with our experiment.

The effective magnetic anisotropy constant $K_{\text {eff }}$ for random assembly of uniaxial or cubic anisotropy nanoparticles can be determined from the law of approach to saturation $M$ [21]. It can be shown that for magnetic susceptibility the 
following equation is valid:

$$
\chi=\frac{\partial M}{\partial H} \cong \frac{\alpha K_{e f f}^{2}}{M_{s} H^{3}}
$$

where $\alpha=0.533$ for uniaxial anisotropy and 0.152 for cubic anisotropy. In Fig. 6 dependence of magnetic susceptibility $\chi$ on inversed cube of magnetic field $H$ for P2 (upper panel) and P1 (lower panel) samples is presented. $K_{\text {eff }}$ can be evaluated from the slope of the straight line that is the leastsquares fit to the experimental points. Assuming $\alpha=0.533$ and taking $M_{s}$ obtained from hysteresis loop, the following values of the effective magnetic anisotropy constant were obtained from equation 3: $K_{\text {eff }}=1.67 \cdot 10^{6} \mathrm{erg} / \mathrm{cc}$ and $2.46 \cdot 10^{6} \mathrm{erg} / \mathrm{cc}$ for P1 and P2 samples, respectively. The obtained values of $K_{\text {eff }}$ are similar to that obtained for bccFe nanoparticles with diameters in $3-40 \mathrm{~nm}$ range prepared by a vapour condensation process [20].

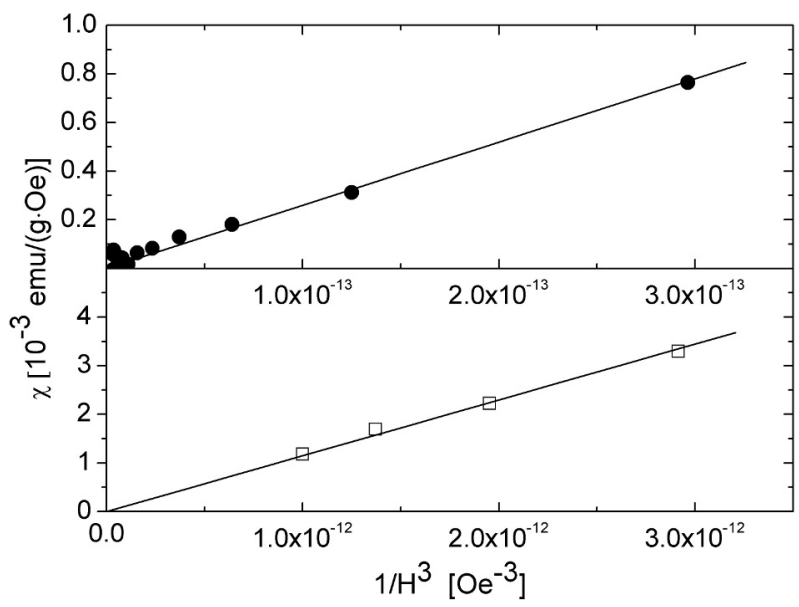

Fig. 6. Dependence of magnetic susceptibility $\chi$ on inversed cube of magnetic field $H^{-3}$ in P2 (upper panel) and P1 (lower panel) samples. The straight line is the least-squares fit to the shown experimental data.

The knowledge of the anisotropy constant $K_{\text {eff }}$ and the saturation magnetization $M_{S}$, for the case of uniaxial anisotropy, enables calculation of the anisotropy field $H_{a}$ with the equation [22]:

$$
H_{a}=\frac{2 K_{e f f}}{\rho M_{s}}
$$

where $\rho\left(7.87 \mathrm{~g} / \mathrm{cm}^{3}\right)$ is density of the sample. From equation $4, H_{a}$ was calculated as $2.59 \mathrm{kOe}$ and $3.98 \mathrm{kOe}$ for samples $\mathrm{P} 1$ and $\mathrm{P} 2$, respectively. These values of $H_{a}$ scale with the nanoparticles sizes and the effective magnetization $4 \pi M_{\text {eff }}$.

\section{Conclusions}

Four samples of nanocrystalline iron with different sizes distributions were studied by static magnetization and FMR spectroscopy. FMR spectrum was analysed in terms of two (for P1, P2, P3 samples) lines attributed to perpendicular and parallel components resulting from magnetic anisotropy field. It was found that the linewidths of the component lines and resulting anisotropy field scale with the average size of the iron nanoparticles. From the ferromagnetic loops observed in P1 and P2 samples the effective magnetic anisotropy constant was calculated: its value was bigger for the sample with smaller nanoparticles. The values of the uniaxial anisotropy fields determined from hysteresis loop parameters for samples P1 and P2 were consistent with these obtained from FMR experiment, thus, confirming the approach applied in FMR spectra analysis.

\section{References}

[1] Gleiter H., Prog. Mater. Sci., 22 (1990), 223.

[2] Weissmuller K. H., Hempelmann J., Phys. Rev. B, 58 (1998), 687.

[3] Loffler J. F., Wagner W., Kostorz G., Appl. Catal. A-Gen., 140 (2000), 115.

[4] Pelka R., Kiębasa K., Arabczyk W., Cent. Eur. J. Chem., 9 (2011), 240.

[5] ArAbCZyK W., LendZion-Bieluń Z., Wróbel R., Polish patent No. P206909.

[6] Arabczyk W., Wróbel R., Solid State Phenom., 94 (2003), 235.

[7] Pelka R., ArabczyK, W., J. Nanomater, 2013 (2013), 645050.

[8] Bodziony T., Guskos N., TypeK J., Roslaniec Z., Narkiewicz U., Maryniak M., Mater. Sci.Poland, 23 (2005), 1055.

[9] TYPEK J., MARYNiaK M., WozniaK M.J., MATYSIAK H., KURZYDLOWSKI K.J., Mater. Sci.-Poland, 24 (2006), 1067.

[10] Helminiak A., Arabczyk W., Zolnierkiewicz G., Guskos N., TypeK J., Rev. Adv. Mater. Sci., 29 (2011), 166

[11] Kiełbasa K., Pelka R., Arabczyk W., J. Phys. Chem. A, 114 (2010), 4531. 
[12] Pelka R., Glinka P., Arabczyk W., Mater. Sci.Poland, 26 (2008), 349.

[13] Guskos N., Anagnostakis E., Likodimos V., Typek J, Maryniak M., Narkiewicz U., J. Appl. Phys., 97 (2005), 0204304.

[14] Guskos N., Glenis S., TyPek J., ZOLnierkiewiCZ G., Berczynski P., WARdAl K., Guskos A., Sibera D., Moszyński D., LoJKowski W., NARKIEWicz U., Cent. Eur. J. Phys., 10 (2012), 470.

[15] WARdal K., TYPeK J., ZolnierkieWicz G., Guskos N., Narkiewicz U., Sibera D., Eur. Phys. J. Appl. Phys., 62 (2013), 10402.

[16] KITTEL CH., Introduction to solid state physics, Wiley, New York, 1967.

[17] Lu H.M., Zheng W.T., Jiang Q., J. Phys. D Appl. Phys., 40 (2007), 320.
[18] Danan H., Herr A., Meyer A.J.P., J. Appl. Phys., 39 (1968), 669.

[19] Gong W., Li H., Zhao Z., Chen J., J. Appl. Phys., 69 (1991), 5119.

[20] Ibusuki T., Kojima S., Kitakami O., Shimada Y., IEEE T. Magn., 37 (2001), 2223.

[21] Chikazumi S., Physics of ferromagnetism, Oxford, Clarendon Press, 1997

[22] Spasojevic V., Mrakovic A., Perovic M., Kusigerski V., Blanusa J., J. Nanopart. Res., 13 (2011), 763. 